\title{
Economía social e ingresos. Algunas tensiones en su articulación en la implementación del Programa de Microcrédito en la ciudad de San Luis.
}

\section{Social economy and incomes. Some tensions in its articulation in implementing the microcredit program in San Luis' city.}

\author{
Ana Laura Hidalgo **
}

\section{Resumen}

El artículo se propone poner en relación las experiencias de la Economía Social y Solidaria (ESS) y el porcentaje de los ingresos que los sujetos perciben de su actividad emprendedora, a fin de reconocer las tensiones entre ambos. El caso de estudio es el Programa Nacional de Microcrédito para la ESS (CONAMI) en la ciudad de San Luis. Este programa funciona de manera descentralizada en el país por medio de los Centros de Referencia (CdR) del Ministerio de Desarrollo Social de la Nación.

El estudio fue realizado durante el año 2014 y su objeto de estudio fueron algunos de los emprendedores que han accedido al programa durante la operatoria 2012-2013. Se realizó un estudio estadístico a partir de una serie de hipótesis planteadas. Finalmente, el trabajo recupera las tensiones reconocidas en el territorio y las presenta en una posible agenda de la política social para el sector.

\footnotetext{
** Ana Laura Hidalgo es licenciada en Comunicación Social por la Universidad Nacional de San Luis (UNSL). Es Especialista en Gestión Social egresada de la Universidad Nacional de Cuyo (UNCUYO) y maestranda en Política y Planificación Social (UNCUYO). Actualmente, es becaria doctoral de CONICET. Correo electrónico: alhidalgo@unsl.edu.ar y hidalgo.analaura@gmail.com
} 


\section{Abstract}

The article aims to relate the experiences of the Social Solidarity Economics (ESS) and the percentage of income that individuals receive from their entrepreneurship activity in order to recognize the tensions between them. The case study is the Programa Nacional de Microcrédito para la ESS (CONAMI) in San Luis. This is decentralized to the country through the Centros de Referencia (CdR) of the Social Development's Ministry of the Nation.

The study was conducted during 2014 on the entrepreneurs who have entered the program during the operation 20122013. It is a statistical study was based on hypotheses. Finally, the work gets recognized tensions in the country and presents a possible agenda for social policy for the sector.

\section{Palabras clave}

Economía social, ingresos, Programa de Microcrédito

\section{Keywords}

Social economic, income, Microcredit's Program.

\section{El abordaje de una política alimentaria: notas introductorias}

El momento de implementación de las políticas públicas conlleva un grado de complejidad creciente en los territorios, en la medida en que supone necesariamente la interacción estratégica y conflictiva entre actores que tienen distintos valores, visiones e intereses respecto de la temática. En el caso de las políticas sociales, el nivel de conflicto suele ser significativo en tanto se trabaja con determinados grados de vulnerabilidades sociales, económicas o culturales de la ciudadanía. Martínez Nogueira (2007) sostiene que la implementación es la esencia de la política pública en tanto determina el sentido de la intervención estatal, ya que del modo en que aquella se implemente condicionará la capacidad de transformación de la sociedad.

Este trabajo presenta parte de las conclusiones obtenidas en el Trabajo Final de la Especialización en Gestión Social de la Facultad de Ciencias Políticas y Sociales (FCPyS) de la Universidad Nacional de Cuyo (UNCUYO) a fin de repensar la problemática de la implementación de una de las políticas públicas sociales que son descentralizadas por el Centro de Referencia (CdR) del Ministerio de Desarrollo Social de la Nación (MDS) en la provincia de San Luis. El objetivo de este trabajo es explorar la posibilidad de sostenibilidad en el tiempo de las experiencias de microcrédito en San Luis, considerando el grado de ingresos proveniente de los emprendimientos de la 
Economía Social y Solidaria (ESS) para los emprendedores de la ciudad capital, respecto del total de estos. Esto propiciará la construcción de una agenda de la política social que recupere las tensiones identificadas en este territorio en particular.

El trabajo se organiza en cuatro momentos. En primer lugar, se expone el marco conceptual desde el cual se abordó el trabajo de campo. Posteriormente, se presentan las características generales del programa analizado. Luego, se exponen los objetivos del trabajo de campo, la estrategia metodológica construida para el abordaje del territorio y las hipótesis. A continuación, se presentan los resultados obtenidos y algunas de las conclusiones y las tensiones reconocidas en su implementación. Finalmente, se esboza una posible agenda de la política social en materia de ESS para el territorio con una intención prospectiva.

Vale mencionar que las conclusiones obtenidas y los temas registrados en la agenda sugerida no pretenden ser generalizados a todo territorio donde se aplica este programa, sino que se pretende generar una mirada situada sobre él.

\section{Fundamentos teóricos}

Muchos autores sostienen que toda economía es necesariamente social porque deviene de las acciones de los hombres y sus relaciones, y no de efectos de la naturaleza -a pesar de los intentos de Adam Smith para pensar la regulación de la oferta y la demanda por medio de una 'mano invisible'. ${ }^{1}$

Boaventura de Souza Santos explica que el paradigma de la modernidad se asienta en dos pilares: el de la regulación y el de la emancipación, cada uno con tres principios o lógicas. El pilar de la regulación está constituido por el principio del Estado -formulado por Hobbes-, el principio del mercado -con las nociones desarrolladas por Locke y Adam Smith- y el principio de comunidad -Rosseau. "El principio del Estado consiste en la obligación política vertical entre ciudadanos y Estado. El principio del mercado, en la obligación política horizontal individualista y antagónica entre los que participan en él. Y el principio de comunidad, que consiste en la obligación política horizontal solidaria entre los miembros de la comunidad y entre las asociaciones" (De Souza Santos, 2000: 52). Por su parte, el pilar de la emancipación está constituido por las tres lógicas de la racionalidad definidas por Weber, a saber: la racionalidad estéticoexpresiva, la cognitivo-instrumental y la moral-práctica. Boaventura de Sousa Santos señala que el principio de mercado ha colonizado los pilares de la regulación, mientras que la racionalidad cognitivo-instrumental ha cooptado los pilares de la emancipación.

La economía, por tanto, atañe a las relaciones sociales que se despliegan en la resolución material de la vida (la subsistencia de la especie); es un aspecto de la vida política de una civilización, que intenta procurar los medios para garantizar la

\footnotetext{
${ }^{1}$ Para ampliar este punto, ver: La economía política en el debate de las ciencias sociales (2013), compiladores Dr. Julio C. Gambina y Dr. Enrique Elorza. Nueva Editorial Universitaria, UNSL.
} 
reproducción social de la vida, este es fundamentalmente su propósito. Sin embargo, cuando se reflexiona en torno a ella, debe repararse que se despliega en un paradigma moderno que supone una sociedad mercantil -que domina la regulación- con una racionalidad cognitivo-instrumental. Ambas características tensionan el despliegue de modos alternativos de resolución del problema de la subsistencia.

Para dar respuesta al problema de lo económico, Roberto Roitman (2011) señala que en la actualidad conviven tres subsistemas en la producción, distribución y consumo de bienes o servicios: el mercado, el estado, y el de la ESS. Si bien cada uno de estos sistemas coexiste en el espacio y en el tiempo, presentan fluidas relaciones entre sí, lógicas internas particulares y dinámicas de funcionamiento totalmente diferentes.

Diversos elementos contextuales han propiciado parte de la invisibilización de la esfera 'social' en la economía; la creciente globalización de los mercados, la separación entre la producción y la generación de empleo, la desregulación de los mercados, la separación cada vez más pronunciada entre trabajo intelectual y trabajo material son algunos de los factores que han condicionado que, en la actualidad, el crecimiento del Producto Bruto Interno (PBI) no garantice necesariamente la generación de empleo suficiente para la cantidad de personas que intenta incorporarse al sistema.

Ante este proceso expansivo de racionalidad económica individualista -característica del mercado-, emerge de los propios tejidos de la sociedad la necesidad de recuperar intentos colectivos de pequeños emprendedores que buscan revalorizar los vínculos asociativos, la toma de decisiones horizontal a su interior, modos propios de la democracia participativa en sus cotidianeidades. Esto conlleva la construcción de un sistema cuyo eje central sea, no el capital financiero o el flujo de la renta, sino el hombre.

\subsection{Economía Social. Ideas y limitaciones}

Mario Elgue (2003) señala que las sociedades materialmente 'ricas' han permitido su coexistencia con el desempleo, la pobreza y la exclusión. Sin dudas, esto señala que los índices de crecimiento del PBI, o los estudios sobre la pobreza no permiten comprender las realidades complejas que suponen las grandes desigualdades. Si bien América Latina atraviesa una evolución macroeconómica favorable en términos de crecimiento convencional, este crecimiento conserva realidades sociales de máxima desigualdad y trabajo informal (Elgue, 2003).

Rofman y Vásquez Blanco (2011) recuperan el debate acerca de dos modelos económico-sociales que han convivido en disputa: "uno de carácter conservador o de Sociedad de Mercado, de corte económico neoliberal, donde la fuerza del mercado funciona como la principal ordenadora social. El otro, de índole popular y redistribucionista, basado en la Sociedad del Trabajo, guiado por la concepción de que la economía, para crecer y desarrollarse a fin de satisfacer la meta de una digna calidad de vida para todos los habitantes del país, debe basarse en el trabajo como único 
generador de valor, en la activa participación del Estado para respaldar a los que menos poder tienen y en una justa distribución de la riqueza y el ingreso nacional" (Rofman y Vásquez Blanco, 2011: 23-24). En este sentido, la ESS permite esfuerzos de cooperación entre seres humanos en formas alternativas de gestión de la actividad productiva que predomina en el sistema; se procura generar lazos de cooperación, solidaridad en toda la cadena de valor, cuidado y respeto por el ecosistema. De este modo, se opone a los objetivos individualistas del sistema capitalista y del modelo conservador.

El Comité Económico y Social Europeo (CESE) en su dictamen sobre el tema "La economía social en América Latina" (2012) señala que en Latinoamérica la forma que adquiere la ESS resuelve de alguna manera las graves situaciones provenientes de falta de equidad social y económica y la vulneración de derechos fundamentales provenientes de esta situación. Y señala, asimismo, que "es una herramienta clave en la lucha a favor del trabajo decente y la superación de la informalidad socioeconómica, resultando esencial en los procesos de desarrollo local y cohesión social" (CESE, 2012: 2). De este modo, las expresiones de la ESS en la región favorecen la democracia económica y posibilitan el acceso al ejercicio de derechos que suponen un mayor grado de autonomía en los territorios.

En Estudio de caracterización y cuantificación de la Economía Social Argentina (2011) el autor afirma que la ESS considera "aquellas actividades asociativas, emprendimientos y movimientos sociales orientados a la producción, comercialización, financiación y/o consumo de bienes y servicios, que reconocen como eje fundamental la cooperación, la solidaridad y el respeto por el medio ambiente, con gestión democrática y primacía de las personas sobre el capital" (Roitman, 2011: 10). La ESS permite la emergencia de relaciones no salariales entre los trabajadores y/o productores, quienes establecen en el interior de la organización mecanismos participativos para la toma de decisiones. Son propietarios del capital, del producto que realizan y de los beneficios que obtienen de su trabajo, alentando la pluralidad y la democracia económica.

Por otra parte, el informe del CESE (2012) advierte que la ESS no debe instalarse como la economía de la pobreza, sino que debe propiciar el cambio de tendencias con justicia social que permitan luchar contra la desigualdad. Esto supone que no debe formar parte de una estrategia aislada o reducida a sectores en situación de vulnerabilidad, sino que, por el contrario, debiera establecerse como una metodología más expandida y consolidada.

Sin embargo, Hintze (2010) admite que las formas de la ESS en América Latina son aún incipientes como estrategia alternativa al capitalismo. Por tanto, son irrupciones en el sistema que requieren de su consolidación y afianzamiento genuinos y sostenidos en el tiempo. En este sentido, se considera prioritario fomentar diversas capacidades y efectos, para contribuir a un cambio necesario de modelo productivo. 
En el mencionado informe, el CESE advierte acerca de la ausencia de políticas públicas integrales y participativas en la región que permitan la emergencia de diversas formas de ESS, más ricas y masivas. Y, al mismo tiempo, señala que "es imprescindible superar las propuestas sustentadas en meras ayudas económicas directas sin contrapartidas, promoviendo acciones destinadas a resolver el problema de sus fuentes de financiación. Deben abordarse políticas estructurales de interés general que incluyan decisiones en materia de legislación, así como el desarrollo de la educación en innovación y de la capacitación profesional, también en el ámbito universitario. Debe potenciarse la presencia de la ESS en el desarrollo de la protección social mediante los sistemas de salud gestionados con los usuarios. Deben consolidarse auténticas políticas de Estado con vocación de continuidad más allá de los sucesivos cambios gubernamentales" (CESE, 2012: 2).

En la región, se destacan de modo incipiente políticas de Estado que abordan integralmente la temática. Sin embargo, en nuestro país el acceso a la financiación por parte de grandes sectores de la ciudadanía sigue siendo un problema endémico que frena radicalmente el desarrollo con un sentido político. A esta limitante se suma la vacancia de la ESS en la planificación general de la economía a nivel de los Estados y la necesidad de implementar políticas públicas de educación, tal como ha sido la recomendación 193 de la OIT (2002).

José Luis Coraggio (2003) retoma los lineamientos de Karl Polanyi y de Marcel Mauss, entendiendo que las propuestas de cambio no serán transformaciones radicales, sino que, por el contrario, consisten en una transición que va posibilitando el surgimiento de alternativas devenidas de construcciones colectivas y de experiencias innovadoras en los territorios particulares. Este se constituye como el desafío de la ESS en la región para pensar un modelo de desarrollo con sentido político, en los términos en que lo presenta Madoery (2013).

\section{El Programa Nacional de Microcrédito como estrategia de ESS}

Hintze (2010) señala que las políticas públicas establecen las condiciones en las que se debe desarrollar la actividad económica, pero al mismo tiempo la condicionan. Es por ello que las políticas públicas no pueden ser pensadas como neutras, sino que conllevan en sí mismas una postura política del Estado respecto de un problema social; por tanto, son complejas e implican contradicciones en la ciudadanía y asumen un carácter dinámico y cambiante a través del tiempo.

En este sentido, Rotman (2011) señala que el sistema de microcrédito permite aliviar la pobreza y contribuye a transformar las estructuras económicas y sociales de los sectores vulnerables, que se encuentran excluidos del circuito de trabajo formal. Permite el acceso de personas carenciadas a pequeños préstamos mediante esquemas especialmente diseñados para atender sus necesidades y circunstancias particulares. 
Desde este punto de vista, el microcrédito es una herramienta que permite la promoción del desarrollo de los territorios.

Las consideraciones del programa indican la necesidad de situar correctamente al microcrédito como un instrumento y no como un fin en sí mismo; de este modo, se convierte en una poderosa herramienta siempre y cuando se combine adecuadamente con otros instrumentos igualmente importantes, tales como capacitación, asistencia técnica, cuestiones organizativas y gremiales que afectan a los emprendedores. Esto conlleva pensar la implementación del programa holísticamente en articulación con otras políticas.

Por tanto, se entiende por microcrédito a la asistencia crediticia para pequeños emprendedores en el marco de la ESS; es un instrumento dirigido a reforzar la dinámica económica del emprendimiento. Al principio, para fortalecer el capital de trabajo de la unidad económica -materiales, insumos, mercaderías- y en los microcréditos sucesivos, el capital fijo -máquinas y herramientas- para que mejore la productividad de la unidad. Como se mencionó anteriormente, es un procedimiento metodológico que combina el crédito con instancias diversas de participación y capacitación; está destinado a pequeños emprendedores, a los cuales les ofrece un apoyo financiero pequeño inserto en un esquema de garantías flexibles, que les permite compartir de manera conjunta los riesgos de sus integrantes. ${ }^{2}$

\subsection{Consideraciones sobre el Programa de Microcrédito}

La Ley 26117 de "Promoción del Microcrédito para el Desarrollo de la Economía Social" fue sancionada el día 28 de junio de 2006 y promulgada el 17 de julio del mismo año. El microcrédito es concebido como una actividad en la que el emprendedor social es acompañado necesariamente por el Estado para favorecer su sustentabilidad y autonomía.

\subsection{Objetivos del programa}

Entre los objetivos del programa se destacan: la promoción del microcrédito como herramienta de financiamiento de los sectores más vulnerables de la población; la promoción de organismos gubernamentales e instituciones no gubernamentales que trabajen con esta modalidad; el apoyo a las instancias que trabajan con el microcrédito mediante herramientas de fortalecimiento; la capacitación de recursos humanos en la metodología; el ofrecimiento de capacitación y asistencia técnica a los emprendedores;

\footnotetext{
${ }^{2}$ La metodología contempla que los emprendedores deben estar insertos en grupos solidarios de aproximadamente cinco sujetos. Cada uno de ellos puede desarrollar su propia actividad y reciben individualmente sus créditos. Pero cada grupo solidario constituye una garantía solidaria al interior de los miembros, quienes deben cubrir el préstamo del grupo en caso de que no pudieren afrontar la devolución pautada.
} 
un nivel de tasa de interés compatible con el desarrollo de los emprendimientos a los que está dirigido (Ley 26 117).

\subsubsection{Destinatarios}

El programa establece que los destinatarios de estos préstamos son las personas físicas o grupos asociativos de bajos recursos que, en el marco de la ESS, se organicen en torno a la gestión del autoempleo; esta política busca facilitar el desarrollo local y regional por parte de los emprendedores en sus contextos particulares (Ley 26 117).

\subsubsection{Tipos de emprendimientos y destino de los fondos}

Los emprendimientos pueden ser individuales, familiares o asociativos. Los primeros hacen referencia a que solo un sujeto está vinculado con la realización del emprendimiento. Los de tipo familiar son destinados a grupos vinculados entre sí por lazos de sangre u hogar. Finalmente, los emprendimientos asociativos son llevados adelante por grupos de personas que no están ligados por relaciones de sangre $u$ hogar.

Asimismo, los emprendimientos de la ESS que pueden ser financiados por el Programa de Microcrédito son todos aquellos que desarrollen actividades de producción, consumo y comercialización, que se encuentren desempeñando sus labores y que dispongan de escasas condiciones de capital. Por esto, no está destinado a actividades que recién se inician, puesto que demandarían otro tipo de herramientas contempladas en el programa "Manos a la Obra". Consecuentemente, el destino de los fondos del microcrédito es el capital de trabajo de las unidades económicas: insumos y herramientas (Ley 26 117).

\subsubsection{Otorgamiento del microcrédito}

Los microcréditos son gestionados por Organizaciones Ejecutoras (OE), que pueden ser asociaciones civiles, fundaciones, cooperativas, entre otras; quedando excluidas las organizaciones comerciales o con fines de lucro. Estas están relacionadas con los Consorcios de Gestión Local y con las Redes de Gestión Asociada que constituyen una plataforma de gestión de los fondos en el territorio (Ley 26 117).

\subsubsection{Garantías y responsabilidades de los actores}

Para acceder al Programa de Microcrédito no son necesarias garantías reales, tales como hipotecas, prendas o recibos de sueldo. Las garantías requeridas por las $\mathrm{OE}$ en el marco de este programa son de tipo solidarias o de ayuda mutua y se constituyen en el seno de los grupos de trabajo que reúnen cinco emprendedores (Grupos solidarios) (Ley 26 117). 


\section{Objetivos, hipótesis y estrategia metodológica del trabajo de campo}

En este apartado, se presentan los objetivos del trabajo de campo, las hipótesis formuladas y la estrategia metodológica con la cual fueron abordados. Asimismo, en ella se explicitan las técnicas de recolección y de procesamiento de los datos obtenidos.

\subsection{Objetivos}

El trabajo de campo se realizó con el propósito de conocer las características sociodemográficas de los sujetos de derecho que accedieron al programa en la ciudad de San Luis durante la operatoria 2012-2013. Posteriormente, se propuso analizar en qué medida los emprendimientos productivos aportan a la economía familiar de los emprendedores (nivel de ingresos proveniente de la actividad). En el presente trabajo se exponen los resultados obtenidos que intentan abordar el segundo de los objetivos mencionados.

\subsection{Hipótesis}

El trabajo de campo estuvo guiado por una serie de hipótesis que constituyen un sistema de anticipaciones de sentidos desprendidas de ciertas inferencias previas. Estas fueron recuperadas especialmente de las tablas de contingencia o cruzadas generadas por el software utilizado para el procesamiento de los datos. Algunas de las relaciones inferidas permitieron vincular el porcentaje de ingresos con: la edad y el nivel de instrucción de los emprendedores, las características del emprendimiento, el tipo de actividad, el tamaño del grupo familiar de convivencia y los beneficiarios del Monotributo Social Costo Cero (MSCC). Algunos de estos datos se presentan en este trabajo.

\subsection{Estrategia metodológica}

La metodología aplicada es de tipo cuantitativa, con propósitos exploratorios, descriptivos y analíticos; tiene por objeto recolectar datos a partir de los cuales analizar e interpretar los fenómenos y sus relaciones. Se trata de una investigación retrospectiva y transversal. Asimismo, la investigación procuró describir la frecuencia y la distribución de los fenómenos en una población o en sus diferentes segmentos, y desprender de esto interpretaciones.

\subsubsection{Muestra}

La muestra estuvo constituida por 112 emprendedores que formaron parte del programa durante el periodo 2012-2013 en la ciudad de San Luis y que constituyen el $82 \%$ de los créditos administrados por la OE "Nueva Sociedad" de esa ciudad. Si bien allí funciona una segunda OE llamada "Caldén", esta concentra la mayoría de sus emprendedores en el interior de la provincia (más del 87\%).

\subsubsection{Técnica e instrumento de recolección de datos}


Las técnicas utilizadas en la presente investigación fueron la encuesta y el análisis documental de la reglamentación del programa y de la bibliografía consultada.

Asimismo, los instrumentos que se utilizaron fueron cuestionarios cerrados presentados a los emprendedores de la ESS.

Se aplicó un cuestionario diferenciado de acuerdo a las actividades que los emprendedores desarrollan, las cuales podrían ser de producción, servicios o comercialización. Las preguntas formuladas indagaron acerca de seis aspectos generales: los datos familiares de los emprendedores; la relación familia-comunidad; las particularidades del emprendimiento; el tipo de administración desempeñada; datos del mercado y el acceso a la formalidad de la actividad.

\subsubsection{Técnica de procesamiento de datos}

Los datos de la variable de estudio, volcados en soporte magnético, fueron analizados mediante la versión actualizada del programa informático Statistic Package for Social Science (software estadístico SPSS) y ordenados en tablas frecuenciales simples y de contingencia que facilitaron el entrecruzamiento de variables. Los análisis se hicieron conforme a los datos estadísticos posibles de calcular (medidas de tendencia central, de variabilidad pruebas de hipótesis, etc.) según lo permitiesen las escalas en que fueron medidas las variables bajo estudio. La interpretación de los datos se hizo teniendo en cuenta el marco teórico, las hipótesis planteadas y los objetivos propuestos para el trabajo.

\section{Resultados obtenidos}

Los datos obtenidos del proceso de investigación se organizaron en las mencionadas tablas, cada una de ellas se presentó con su correspondiente gráfico y notas aclaratorias que orientan su lectura y comprensión. A continuación, se presentan algunas de las tablas de contingencia obtenidas del proceso de investigación en el territorio, de acuerdo a las hipótesis descriptas anteriormente.

Tabla n. ${ }^{\text {ro }} 1$. "Distribución de los emprendedores según nivel de instrucción y el porcentaje de ingresos provenientes de la actividad del microcrédito con relación al total de los ingresos"

\begin{tabular}{|c|c|c|c|c|c|c|c|c|c|c|c|c|}
\hline \multirow{3}{*}{$\begin{array}{c}\text { Distribución de los } \\
\text { encuestados según } \\
\text { el nivel de } \\
\text { instrucción }\end{array}$} & \multicolumn{10}{|c|}{$\begin{array}{l}\text { Distribución de los encuestados según porcentaje de } \\
\text { sus ingresos }\end{array}$} & \multirow{2}{*}{\multicolumn{2}{|c|}{ Total }} \\
\hline & \multicolumn{2}{|c|}{$\begin{array}{c}\text { Sin } \\
\text { datos }\end{array}$} & \multicolumn{2}{|c|}{$25 \%$} & \multicolumn{2}{|c|}{$50 \%$} & \multicolumn{2}{|c|}{$75 \%$} & \multicolumn{2}{|c|}{$100 \%$} & & \\
\hline & $f$ & $\%$ & $f$ & $\%$ & $\mathrm{~F}$ & $\%$ & $f$ & $\%$ & $f$ & $\%$ & $f$ & $\%$ \\
\hline Sin datos & 3 & 2,67 & 0 & 0 & 0 & 0 & 0 & 0 & 0 & 0 & 3 & 2,67 \\
\hline $\begin{array}{l}\text { Primario } \\
\text { Incompleto }\end{array}$ & 0 & 0 & 1 & 0,89 & 0 & 0 & 0 & 0 & 2 & 1,78 & 3 & 2,67 \\
\hline
\end{tabular}




\begin{tabular}{|c|c|c|c|c|c|c|c|c|c|c|c|c|}
\hline $\begin{array}{l}\text { Primario } \\
\text { Completo }\end{array}$ & 1 & 0,89 & 9 & 8,03 & 12 & 10,71 & 2 & 1,78 & 5 & 4,46 & 29 & 25,89 \\
\hline $\begin{array}{l}\text { Secundario } \\
\text { Incompleto }\end{array}$ & 2 & 1,78 & 13 & 11,60 & 12 & 10,71 & 5 & 4,46 & 8 & 7,14 & 40 & 35,71 \\
\hline $\begin{array}{l}\text { Secundario } \\
\text { Completo }\end{array}$ & 1 & 0,89 & 5 & 4,46 & 4 & 3,57 & 4 & 3,57 & 7 & 6,25 & 21 & 18,75 \\
\hline $\begin{array}{c}\text { Terciario } \\
\text { Incompleto }\end{array}$ & 0 & 0 & 2 & 1,78 & 2 & 1,78 & 3 & 2,67 & 1 & 0,89 & 8 & 7,14 \\
\hline $\begin{array}{l}\text { Terciario } \\
\text { Completo }\end{array}$ & 0 & 0 & 1 & 0,89 & 2 & 1,78 & 1 & 0,89 & 0 & 0 & 4 & 3,57 \\
\hline $\begin{array}{l}\text { Universitario } \\
\text { Incompleto }\end{array}$ & 0 & 0 & 1 & 0,89 & 2 & 1,78 & 1 & 0,89 & 0 & 0 & 4 & 3,57 \\
\hline Total & 7 & 6,25 & 32 & 28,57 & 34 & 30,35 & 16 & 14,28 & 23 & 20,53 & 112 & 100 \\
\hline
\end{tabular}

Fuente: Hidalgo, A. (2014). Análisis de la Implementación de CONAMI en la ciudad de San Luis. Aproximaciones a las problemáticas de la ejecución de Políticas Sociales ${ }^{3}$

La tabla n. ${ }^{\text {ro }} 1$ vincula el nivel de instrucción de los emprendedores de la ESS de la ciudad de San Luis con el nivel de ingresos que perciben de sus emprendimientos. Tal como lo demuestran los niveles expresados, en esta tabla se ha obtenido la máxima asociación posible $(p=0,000)$.

Se observa que un $11,6 \%$ del total de los encuestados posee el secundario incompleto y obtienen un $25 \%$ de sus ingresos con el emprendimiento.

Por su parte, el $10,71 \%$ del total de los trabajadores consultados posee el nivel primario completo y alcanza la mitad de sus ingresos (50\%) con el emprendimiento; el mismo nivel es alcanzado por otro $10,71 \%$ que ha transitado el nivel secundario de modo incompleto.

Un pequeño grupo, que representa el 4,46\% del total, alcanza el $75 \%$ de los ingresos y tiene el nivel secundario incompleto. Finalmente, el 6,25\% tiene el nivel secundario completo y logra alcanzar el $100 \%$ de sus ingresos con el emprendimiento.

Como se observa, existe una importante vinculación entre el nivel de instrucción alcanzado por los emprendedores y los niveles de ingresos que perciben por parte de los proyectos productivos.

\footnotetext{
3 Hidalgo, A. (2014). Análisis de la Implementación de CONAMI en la ciudad de San Luis. Aproximaciones a las problemáticas de la ejecución de Políticas Sociales (2014). Tesina de Especialización en Gestión Social, Facultad de Ciencias Políticas y Sociales, Universidad Nacional de Cuyo (Inédito).
} 
Tabla n. ${ }^{\text {ro }}$ 2. "Distribución de los emprendedores según características del emprendimiento y el porcentaje de ingresos provenientes de la actividad del microcrédito con relación al total de los ingresos"

\begin{tabular}{|c|c|c|c|c|c|c|c|c|c|c|}
\hline \multirow{3}{*}{$\begin{array}{l}\text { Distribución de los } \\
\text { encuestados según } \\
\text { porcentaje de sus ingresos }\end{array}$} & \multicolumn{8}{|c|}{$\begin{array}{l}\text { Distribución de los encuestados según el } \\
\text { emprendimiento }\end{array}$} & \multirow{2}{*}{\multicolumn{2}{|c|}{ Total }} \\
\hline & \multicolumn{2}{|c|}{$\begin{array}{l}\text { Sin } \\
\text { datos }\end{array}$} & \multicolumn{2}{|c|}{ Individual } & \multicolumn{2}{|c|}{ Asociativo } & \multicolumn{2}{|c|}{ Familiar } & & \\
\hline & $\mathrm{F}$ & $\%$ & $f$ & $\%$ & $f$ & $\%$ & $\mathrm{~F}$ & $\%$ & $f$ & $\%$ \\
\hline Sin datos & 0 & 0 & 6 & 5,35 & 0 & 0 & 1 & 0,89 & 7 & 6,25 \\
\hline $25 \%$ & 1 & 0,89 & 23 & 20,53 & 3 & 2,67 & 5 & 4,46 & 32 & 28,57 \\
\hline $50 \%$ & 1 & 0,89 & 27 & 24,10 & 1 & 0,89 & 5 & 4,46 & 34 & 30,35 \\
\hline $75 \%$ & 0 & 0 & 12 & 10,71 & 0 & 0 & 4 & 3,57 & 16 & 14,28 \\
\hline $100 \%$ & 1 & 0,89 & 13 & 11,60 & 3 & 2,67 & 6 & 5,35 & 23 & 20,53 \\
\hline Total & 3 & 2,67 & 81 & 72,32 & 7 & 6,25 & 21 & 18,75 & 112 & 100 \\
\hline
\end{tabular}

Fuente: Hidalgo, A. (2014) Análisis de la Implementación de CONAMI en la ciudad de San Luis. Aproximaciones a las problemáticas de la ejecución de Políticas Sociales

La tabla n. $^{\text {ro }} 2$ vincula los niveles de ingreso percibidos a través del emprendimiento de la ESS con sus características, pudiendo ser individuales, asociativas o familiares (ver 3.1.3).

La mayoría de los emprendimientos de la ESS son de carácter individual, representando el 72,32\% de los encuestados. Por su parte, el 6,25\% trabaja en los de tipo asociativo y el $18,75 \%$ está involucrado en emprendimientos familiares.

Dentro de los emprendimientos de tipo individual, el $24,10 \%$ de los emprendedores alcanzan el $50 \%$ de sus ingresos. Un $20,53 \%$ de ellos obtiene el $25 \%$ de sus requerimientos. Por su parte, el $11,60 \%$ del total de los encuestados alcanza el $100 \%$ de sus ingresos con el emprendimiento.

Los niveles de emprendimientos asociativos son claramente más bajos; solo el 2,67\% de estos alcanzan el $100 \%$ de sus ingresos con la actividad. En cuanto a los emprendimientos de carácter familiar, se destaca que el 5,35\% del total obtiene del emprendimiento el $100 \%$ de sus requerimientos.

Esto refuerza la idea de que la modalidad más extendida entre los emprendedores es la individual, a pesar de las consideraciones del programa. 
Tabla n. ${ }^{\text {ro }} 3$. "Distribución de los emprendedores según tipo de actividad y el porcentaje de ingresos provenientes de la actividad del microcrédito con relación al total de los ingresos"

\begin{tabular}{|c|c|c|c|c|c|c|c|c|}
\hline \multirow{3}{*}{$\begin{array}{c}\text { Distribución de los } \\
\text { encuestados según porcentaje } \\
\text { de sus ingresos }\end{array}$} & \multicolumn{6}{|c|}{$\begin{array}{l}\text { Distribución de los encuestados según la } \\
\text { actividad/oficio }\end{array}$} & \multirow{2}{*}{\multicolumn{2}{|c|}{ Total }} \\
\hline & \multicolumn{2}{|c|}{ Servicio } & \multicolumn{2}{|c|}{ Producción } & \multicolumn{2}{|c|}{ Comercialización } & & \\
\hline & $f$ & $\%$ & $f$ & $\%$ & $f$ & $\%$ & $f$ & $\%$ \\
\hline Sin datos & 0 & 0 & 4 & 3,57 & 3 & 2,67 & 7 & 6,25 \\
\hline $25 \%$ & 0 & 0 & 27 & 24,10 & 5 & 4,46 & 32 & 28,57 \\
\hline $50 \%$ & 4 & 3,57 & 25 & 22,32 & 5 & 4,46 & 34 & 30,35 \\
\hline $75 \%$ & 3 & 2,67 & 11 & 9,82 & 2 & 1,78 & 16 & 14,28 \\
\hline $100 \%$ & 5 & 4,46 & 16 & 14,28 & 2 & 1,78 & 23 & 20,53 \\
\hline Total & 12 & 10,71 & 83 & 74,10 & 17 & 15,17 & 112 & 100 \\
\hline
\end{tabular}

Fuente: Hidalgo, A. (2014). Análisis de la implementación de CONAMI en la ciudad de

San Luis. Aproximaciones a las problemáticas de la ejecución de políticas sociales

En la tabla n. $^{\text {ro }} 3$ se vinculan el porcentaje de ingresos de los emprendedores y el tipo de actividad que realizan.

El 74,10\% de los consultados realiza actividades de producción; el 15,17\% desempeña actividades de comercialización $y$, por su parte, el 10,71\% desempeña tareas de servicio.

Las actividades de producción son las más escogidas por los emprendedores de la ESS. Un $14,28 \%$ de los emprendedores que desarrollan este tipo de actividades alcanza a obtener el $100 \%$ de sus ingresos con el emprendimiento. En menor medida, pero con un porcentaje también destacado, los emprendedores que realizan estas actividades alcanzan el $50 \%$ y el $25 \%$ de sus ingresos representado por un $22,32 \%$ y un $24,10 \%$ del total, respectivamente.

Por otro lado, solo el $4,46 \%$ de los emprendedores que realizan actividades de servicio alcanzan el $100 \%$ de sus ingresos con la actividad; y el 1,78\% lo hace realizando tareas de comercialización. 
Tabla n. ${ }^{\text {ro }}$ 4. "Distribución de los encuestados según el tamaño del grupo familiar conviviente y el porcentaje de ingresos provenientes de la actividad del microcrédito con relación al total de los ingresos"

\begin{tabular}{|c|c|c|c|c|c|c|c|c|c|c|c|c|}
\hline \multirow{3}{*}{$\begin{array}{l}\text { Distribución de los } \\
\text { encuestados según } \\
\text { tamaño del grupo } \\
\text { familiar conviviente }\end{array}$} & \multicolumn{10}{|c|}{$\begin{array}{l}\text { Distribución de los encuestados según porcentaje de } \\
\text { sus ingresos }\end{array}$} & \multirow{2}{*}{\multicolumn{2}{|c|}{ Total }} \\
\hline & \multicolumn{2}{|c|}{$\begin{array}{c}\text { Sin } \\
\text { datos }\end{array}$} & \multicolumn{2}{|c|}{$25 \%$} & \multicolumn{2}{|c|}{$50 \%$} & \multicolumn{2}{|c|}{$75 \%$} & \multicolumn{2}{|c|}{$100 \%$} & & \\
\hline & $f$ & $\%$ & $f$ & $\%$ & $f$ & $\%$ & $\mathrm{~F}$ & $\%$ & $f$ & $\%$ & $f$ & $\%$ \\
\hline, 00 & 0 & 0 & 1 & 0,89 & 3 & 2,67 & 0 & 0 & 0 & 0 & 4 & 3,57 \\
\hline 1,00 & 0 & 0 & 0 & 0 & 1 & 0,89 & 0 & 0 & 0 & 0 & 1 & 0,89 \\
\hline 2,00 & 0 & 0 & 3 & 2,67 & 7 & 6,25 & 1 & 0,89 & 3 & 2,67 & 14 & 12,5 \\
\hline 3,00 & 1 & 0,89 & 7 & 6,25 & 4 & 3,57 & 5 & 4,46 & 12 & 10,71 & 29 & 25,89 \\
\hline 4,00 & 5 & 4,46 & 9 & 8,03 & 10 & 8,92 & 6 & 5,35 & 2 & 1,78 & 32 & 28,57 \\
\hline 5,00 & 1 & 0,89 & 7 & 6,25 & 4 & 3,57 & 2 & 1,78 & 2 & 1,78 & 16 & 14,28 \\
\hline 6,00 & 0 & 0 & 3 & 2,67 & 4 & 3,57 & 1 & 0,89 & 3 & 2,67 & 11 & 9,82 \\
\hline 7,00 & 0 & 0 & 1 & 0,89 & 0 & 0 & 0 & 0 & 1 & 0,89 & 2 & 1,78 \\
\hline 8,00 & 0 & 0 & 1 & 0,89 & 1 & 0,89 & 1 & 0,89 & 0 & 0 & 3 & 2,67 \\
\hline Total & 7 & 6,25 & 32 & 28,57 & 34 & 30,35 & 16 & 14,28 & 23 & 20,53 & 112 & 100 \\
\hline
\end{tabular}

Fuente: Hidalgo, A. (2014). Análisis de la implementación de CONAMI en la ciudad de San Luis. Aproximaciones a las problemáticas de la ejecución de políticas sociales

La tabla n. ${ }^{\text {ro }} 4$ relaciona el porcentaje de ingresos de los emprendedores prevenientes de su actividad y el número de personas que integran el grupo familiar de convivencia. Un $28,57 \%$ de los emprendedores conviven en grupos familiares integrados por cuatro personas y el $25,89 \%$ lo hace en grupos de solo tres individuos. El $2,67 \%$ de los consultados convive con ocho personas.

Un grupo que representa el $8,03 \%$ de los consultados alcanza solo el $25 \%$ de sus ingresos y convive con cuatro personas. Un 8,92\% obtiene el $50 \%$ de sus ingresos con el emprendimiento y pertenece a un grupo familiar integrado también por cuatro personas. Alcanzan el 75\% de los ingresos requeridos el 5,35\% de los encuestados que conviven también con cuatro personas. Un 10,71\% alcanza el $100 \%$ de sus ingresos con el emprendimiento y pertenecen a familias integradas por tres miembros.

De lo anterior se destaca que los emprendedores que obtienen niveles altos de ingresos con la actividad, pertenecen a grupos de convivencia pequeños. Esto permite inferir el tamaño y las características de los emprendimientos, en relación con su capacidad real para dar respuesta y afrontar las necesidades de los grupos familiares. En algunos casos, estos índices insuficientes para afrontar los costos del grupo de 
convivencia se ven compensados con la existencia de otros trabajos por parte de los emprendedores o bien por otro miembro del grupo de convivencia.

Tabla n. ${ }^{\text {ro }}$ 5. "Distribución de los emprendedores según inscripción en Monotributo Social Costo Cero y el porcentaje de ingresos provenientes de la actividad del microcrédito con relación al total de los ingresos"

\begin{tabular}{|c|c|c|c|c|c|c|c|c|}
\hline \multirow{3}{*}{$\begin{array}{c}\text { Distribución de los } \\
\text { encuestados según } \\
\text { porcentaje de sus ingresos }\end{array}$} & \multicolumn{6}{|c|}{$\begin{array}{l}\text { Distribución de los encuestados según si está } \\
\text { inscripto en el Monotributo Social Costo Cero }\end{array}$} & \multirow{2}{*}{\multicolumn{2}{|c|}{ Total }} \\
\hline & \multicolumn{2}{|c|}{ Sin datos } & \multicolumn{2}{|c|}{ Sí } & \multicolumn{2}{|c|}{ No } & & \\
\hline & $f$ & $\%$ & $f$ & $\%$ & $f$ & $\%$ & $f$ & $\%$ \\
\hline Sin datos & 3 & 2,67 & 0 & 0 & 4 & 3,57 & 7 & 6,25 \\
\hline $25 \%$ & 6 & 5,35 & 1 & 0,89 & 25 & 22,32 & 32 & 28,57 \\
\hline $50 \%$ & 2 & 1,78 & 2 & 1,78 & 30 & 26,78 & 34 & 30,35 \\
\hline $75 \%$ & 2 & 1,78 & 2 & 1,78 & 12 & 10,71 & 16 & 14,28 \\
\hline $100 \%$ & 5 & 4,46 & 3 & 2,67 & 15 & 13,39 & 23 & 20,53 \\
\hline Total & 18 & 16,07 & 8 & 7,14 & 86 & 76,78 & 112 & 100 \\
\hline
\end{tabular}

Fuente: Hidalgo, A. (2014) Análisis de la implementación de CONAMI en la ciudad de

San Luis. Aproximaciones a las problemáticas de la ejecución de políticas sociales

La tabla n. ${ }^{\text {ro }} 5$ presenta los datos cruzados en relación con los niveles de ingreso de los emprendedores y su suscripción en el Monotributo Social Costo Cero (MSCC).

En ella se observa que solo un 7,14\% se encuentra inscripto; de los cuales un $2,67 \%$ obtiene el $100 \%$ de los ingresos destinados a su subsistencia. Un 1,78\% alcanza las tres cuartas partes y el mismo porcentaje llega a la mitad de sus ingresos con la actividad desempeñada en el emprendimiento.

Un 76,78\% no está inscripto en el MSCC. De este grupo, un 26,78\% alcanza la mitad de sus requerimientos en ingresos; un 22,32\% solo se aproxima a un cuarto de lo que necesitaría para poder vivir únicamente de su emprendimiento. Por su parte, un $10,71 \%$ alcanza el $75 \%$ y el $13,39 \%$ la totalidad de sus requerimientos.

De lo anterior se desprende que el grado de informalidad de los emprendedores de la ESS es muy significativo. Aun los emprendimientos que alcanzan un alto nivel de ingresos de acuerdo con sus requerimientos conservan su informalidad. Esto permite problematizar los sentidos acerca del ejercicio del derecho al trabajo por parte de los emprendedores de la ESS. 


\section{Algunas tensiones emergentes del caso de estudio. Primeras conclusiones}

Este trabajo ha presentado una aproximación descriptiva a la problemática de la implementación del Programa Nacional de Microcrédito para la ESS en la ciudad de San Luis; vale mencionar que se han incluido solo cinco tablas de las cincuenta y ocho confeccionadas a partir del trabajo de campo. A continuación, se presentan las principales ideas que se desprenden de este estudio exploratorio sobre los sujetos, la política y el territorio, para luego esbozar los intentos de una agenda de la política social con clave situada. ${ }^{4}$

Los emprendimientos de la ESS de la ciudad de San Luis son desempeñados por adultos de mediana edad que no acceden al empleo formal; consecuentemente, el porcentaje de los emprendedores menores de 20 años y mayores de 50 años no es significativo. En cuanto al nivel de instrucción, se señala que el $67 \%$ no ha terminado sus estudios secundarios, lo que indica que se trata de sujetos a los cuales se les dificulta el acceso a un empleo calificado o estable. Existe una importante asociación entre el nivel de instrucción de los emprendedores y el nivel de ingresos que perciben de sus emprendimientos, tal como se ha advertido.

Los emprendimientos son mayormente desempeñados por mujeres $(78,6 \%)$, quienes consiguen adaptarse con mejores resultados a la modalidad del programa, puesto que les permite la articulación de este con las tareas de reproducción de la vida cotidiana. Esto podría deberse a que, en general, los hombres ocupan su fuerza de trabajo en otras modalidades de trabajo formal o informal.

Los emprendedores componen grupos familiares numerosos, compuestos por más de tres integrantes. El 87,5\% contribuye con su actividad a la economía familiar, por lo cual se sostiene el alto impacto que conserva el programa en las familias y en los circuitos de la ESS.

Los emprendimientos son predominantemente individuales $(72,3 \%)$, y los fondos son destinados a la producción (74,1\%). En los emprendimientos de la ESS no predominan los de tipo asociativo, a pesar de que esto es expresamente señalado en sus fundamentos. Por el contrario, prevalecen lógicas de trabajo tradicional o de relaciones sociales propias de las economías de mercado capitalista; las relaciones sociales se encuentran determinadas por el mercado (De Souza Santos, 2003) y este es el escenario que rodea el ámbito de ejercicio de los trabajadores de la ESS. Si bien la experiencia del microcrédito es significativa para pensar en otras relaciones humanas, debe acompañarse por otras prácticas que permitan la re-socialización de los ciudadanos en estos sentidos.

La operatoria 2012-2013 está compuesta por un grupo de emprendedores nuevos en la dinámica del programa (56,3\%). Asimismo, el 83,9\% no participa de otro programa

\footnotetext{
${ }^{4}$ En este punto, se presentan otros datos obtenidos del trabajo de campo que no han podido ser expuestos en las tablas anteriores por cuestiones de espacio.
} 
provincial o municipal; el microcrédito ha llegado a sectores que anteriormente no habían podido acceder. Por su parte, el 10,7\% manifestó acceder a otros programas, de los cuales se destaca con un 3,6\% de estos, el Plan de Inclusión Social dependiente del Estado provincial de San Luis.

El 33\% de los emprendedores manifiesta no tener conocimiento acerca de otros programas nacionales y el $44,6 \%$ dice no acceder a ellos. Esto representa un importante número de familias que se siente fuera de las convocatorias. Sin embargo, en esto también se destaca que no se sienten parte de las políticas públicas sociales, a pesar de ser emprendedores de la ESS dependiente del Ministerio de Desarrollo Social de la Nación. Entre los motivos expresados se destaca cierta falta de conocimiento acerca de su funcionamiento.

Los ingresos provenientes de los emprendimientos no son suficientes para afrontar los requerimientos de las familias. Esto también implica que los sujetos no pueden dedicarse absolutamente a una actividad productiva. El 31,3\% tiene otro trabajo en relación de dependencia, esto indica la presencia de sobreocupación de los emprendedores de la ESS. Solo el 20,5\% de los emprendedores cubre la totalidad de sus requerimientos con los ingresos provenientes del emprendimiento.

Los emprendedores que obtienen niveles altos de ingresos con la actividad pertenecen a grupos de convivencia pequeños, lo que indica que los ingresos provenientes de los emprendimientos son escasos o incipientes.

La variación en horas dedicadas al emprendimiento en relación con los ingresos obtenidos es muy relativa; la mayoría de los emprendedores de la ESS no dedican sus horas productivas solo al desarrollo de la actividad. Esto se debe a que los sujetos desarrollan otras actividades productivas simultáneamente; por esto, los ingresos provenientes del emprendimiento son insuficientes.

Un grupo muy pequeño de emprendedores de la ESS de la ciudad de San Luis ha accedido a la modalidad de MSCC, representando el 76,8\%; un amplio sector de los trabajadores no cuenta con las garantías y derechos de todo trabajador. Entre los motivos manifestados, el 11,6\% manifestó adeudar el crédito; el 7,1\% indicó que desconocía acerca del MSCC y, con el mismo porcentaje, otros trabajadores indican que el emprendimiento es aún chico o ha iniciado la actividad recientemente. Muchos no acceden al MSCC por no reunir las condiciones o bien por el desconocimiento de la modalidad.

Por su parte, solo el 8,9\% de los emprendedores accedió a la obra social, lo que refuerza la situación de precariedad laboral del sector. Entre los motivos, se destaca que los sujetos desconocen los mecanismos. Del mismo modo, el grupo de emprendedores que da cumplimiento a los requerimientos de Ingresos Brutos es muy bajo y poco representativo, siendo solo el $8 \%$. Son muy pocos los emprendedores que manifiestan haber iniciado el trámite $(3,6 \%)$. 
Solo el $0,9 \%$ de los trabajadores tiene regularizada su situación respecto de los impuestos y requisitos municipales; lo cual refuerza la idea que los trabajadores de la ESS no poseen garantías laborales adecuadas para el desarrollo de sus actividades y el ejercicio de sus derechos. El trabajo de los emprendedores se caracteriza por su informalidad.

Finalmente, se puede señalar que, de la implicación de las actividades de la ESS y la satisfacción de los requerimientos familiares en la ciudad de San Luis, se desprenden, al menos, los siguientes factores:

- Se reconoce un impacto relativamente bajo en las familias intervinientes, en los términos en los cuales han sido considerados en este trabajo. ${ }^{5}$ Países como Ecuador, México ("Primer Empleo"), o Perú registran un mayor nivel de impacto en políticas sociales de este tipo.

- El Estado provincial debiera reconocer en su legislación a los sujetos de la ESS, a sabiendas de que son sujetos que acceden sin garantías o patrimonios importantes. Es difícil pensar en su responsabilidad a la contribución para la redistribución -tomando de este modo los impuestos-, cuando sus actividades económicas requieren ser promocionadas por el Estado.

\section{Algunas discusiones finales a incluir en una agenda de la política social}

De acuerdo con el modelo y tendencias que propone el programa analizado, se puede afirmar que conserva ciertas particularidades en las que el Estado aparece como un agente con una intervención importante en materia de regulaciones al sector, asimilándose al modelo popular redistribucionista (Rofman y Vásquez Blanco, 2011). De este modo, se destaca una fuerte presencia del Estado y una pequeña participación del sector privado. Sin embargo, en la actualidad se advierte que el trabajo ha perdido la centralidad para garantizar los derechos sociales, puesto que se consolida una relación transferencia de dinero y trabajo.

En materia social, se ha consolidado el mercado de trabajo informal; en lo político se habla de una 'crisis de representación' y en lo cultural se manifiesta cierta 'crisis de ideologías'. Por todo esto, se advierten ciertas rupturas en el caso de estudio con el llamado 'Estado neoliberal' (flexibilización laboral, desempleo, trabajo informal, crisis de representación, acción individual, crisis ideológica, entre otras). Sin embargo, aún se evidencia la recuperación de un 'Estado social' de modo muy lejano (en contraposición al anterior), puesto que sus principales características (pleno empleo, mercado interno en expansión, regulación importante de la economía, trabajador como sujeto social, lo político como constitutivo de la identidad y predominio de la acción colectiva, entre

\footnotetext{
${ }^{5}$ Se considera que, en Argentina, unas 200 mil personas acceden al Programa Nacional de Microcrédito para la Economía Social y Solidaria (Arroyo, 2014).
} 
otras) no encuentran en la actualidad circunstancias que constituyan condiciones de posibilidad para sostenerse en el tiempo.

Se observa que el número de sujetos que accede al programa es muy bajo y este no es solo un fenómeno de la ciudad de San Luis. Esto es también reconocido por el informe del CESE (2012), en el cual se advierte que no debe ser un mecanismo marginal o destinado a sectores en situación de pobreza. Por el contrario, debiera concebirse en el marco de políticas públicas integrales con mayores niveles de participación -no solo en la gestión del programa-; esto apunta a generar mecanismos de desarrollo local con superiores grados de cohesión social (CESE, 2012). Como advierte Hintze (2010), la ESS no debe ser pensada como estrategia aislada, sino que requiere afianzamiento en el tiempo y fortalecerse como alternativa al capitalismo.

Asimismo, se advierte que esta problemática atraviesa las dificultades que manifiestan los jóvenes para acceder a su primer empleo, o de las personas de mediana edad que han tenido que salir de los circuitos formales de trabajo. De este modo, la estructura social de la sociedad argentina actual manifiesta una paralización de la movilidad descendente característica de los escenarios neoliberales, pero al mismo tiempo no pudo reconstruirse la movilidad ascendente. El Programa de Microcréditos está destinado a los sectores vulnerables de la sociedad que se encuentran en la informalidad económica; vale mencionar que el Estado tiene pocos intentos en esta dirección.

En términos de organización, el Programa de Microcréditos impulsó un tipo mixto de gestión que le permite una bajada en el territorio particular; puesto que articula organizaciones de la sociedad civil, del mercado y del Estado en el abordaje de las problemáticas. Se recupera como eje la cooperación, el respeto al medio ambiente, la solidaridad y una gestión más democrática (Roitman, 2011). Vale mencionar que estos son cambios a largo plazo pero que en la metodología se advierte un desplazamiento de la centralidad del capital hacia las personas. Se advierten pequeños desplazamientos en las relaciones no salariales que se establecen entre sus miembros.

Otro de los temas pendientes en esta agenda de la política social es la necesidad de trabajar en la capacidad de las políticas sociales para generar condiciones de posibilidad para una empleabilidad estable; ese pasaje no ha quedado claro en la letra del programa. Se considera que esto permitiría propiciar una discusión sobre la creciente fragmentación entre los diversos sectores, la desigualdad y la exclusión. Por esto, debe impulsarse un nuevo concepto de desarrollo, ligado a las realidades de los territorios que se habitan (Madoery, 2013). En este sentido, desarrollo no puede ser sinónimo de crecimiento económico, en el cual se destaca el énfasis en los factores explicativos monodisciplinarios, subrayándose de modo especial la variante económica. El nuevo concepto de desarrollo debe enfatizar la identidad cultural y la multidimensionalidad. Algunos de sus principios son: la satisfacción de necesidades básicas para erradicar la pobreza, un proceso endógeno y autónomo, y en armonía con 
el medio ambiente (Servaes, 2000). Desde esta perspectiva no hay un patrón universal de desarrollo y debe ser concebido como un proceso integral, multidimensional y dialéctico que puede diferir de una sociedad a otra.

De lo analizado se desprende, adicionalmente, que es una fuerte necesidad la generación de empleo por medio del Estado de modo directo e indirecto. Esto es, generar mecanismos por los cuales se pueda acceder a formas más estables de vinculación con su mercado y fortalecer el emprendimiento. Para esto, acceder a un lugar fijo de ventas, a créditos más importantes, y, sobre todo, al sistema del MSCC como emprendedores de la ESS. Esta última limitación requiere un estudio más profundo de las causas por las cuales la gran mayoría de los emprendedores no optan por él.

Se requiere analizar el impacto de esta política en términos de diseño, efectividad y sustentabilidad. La posibilidad de incrementar la descentralización de otras políticas sociales abrirá la posibilidad para acercarse a los territorios y, de este modo, fusionar los horizontes de sentido de los protagonistas de las políticas públicas: los mismos actores territoriales. Avanzar hacia un modelo de gestión basado en una 'gestión asociada' implicaría apuntar a la sustentabilidad de la propuesta, ya que conlleva el diseño y la ejecución junto con las organizaciones sociales. Pero este modelo de gestión es inalcanzable si no se recuperan las concepciones de desarrollo que se conciben en los territorios.

La falta de evaluación del programa seleccionado a nivel nacional se suma al problema de los escasos análisis de implementación que conllevan estas experiencias. Se sabe que la dinámica de la gestión muchas veces hace reemplazar lo importante por lo urgente, pero se considera que la falta de seguimiento de la ejecución de las políticas públicas es una de las limitantes mayores a considerar. Por otra parte, sería oportuno que se generase un mecanismo de evaluación y análisis de la política que considere la gran fragmentación territorial de nuestro país, los lugares críticos, las desigualdades constitutivas, entre otros factores.

Asimismo, resultaría propicio que se impulsase con algún mecanismo conveniente la consolidación de una sociedad civil fuerte que permita construir su propia agenda para poder incidir en las agendas políticas y mediáticas. Si bien en nuestro país el financiamiento de este sector no es claro, la presencia territorial de las organizaciones ha disputado el espacio con los partidos políticos tradicionales.

A pesar de que el presente análisis de la implementación del programa no puede generalizarse por los motivos que se mencionaron anteriormente, se concluye a partir de lo expuesto que el Programa de Microcrédito para la ESS en San Luis ha tenido un bajo impacto en términos de masividad; sin embargo, el estudio releva una gran cantidad de potencialidades para el sector, de cara a construir una agenda de la política social 'de segunda generación' (Arroyo, 2014). 
Por otra parte, considerando los escasos antecedentes en materia de evaluación de políticas públicas sociales en este territorio, esta experiencia es alentadora en la medida en que las ausencias dicen tanto como las presencias. La invitación es a revalorizar el momento de la implementación como apertura para añadir valor a las propias políticas.

\section{Referencias bibliográficas}

Abramovich, V. (2006). Una aproximación al enfoque de derechos en las estrategias y políticas de desarrollo. Revista de la CEPAL n 88, 36-50.

Arroyo, D. (2014). Apuntes de clase del Seminario "Agenda de la Política Social". Facultad de Ciencias Políticas y Sociales, Universidad Nacional de Cuyo. (Inédito)

Comité Económico y Social Europeo (CESE). (2012). Dictamen sobre el tema "La Economía Social en América Latina". Ponente: Miguel Ángel Cabra de Luna. REX/325. La Economía Social en América Latina. Bruselas, 22 de febrero de 2012.

Coraggio, J.L. (2003). Presentación para el público latinoamericano en Laville, Jean-Louis (Comp.) Economía Social y Solidaria. Una visión europea. Buenos Aires: UNGS. Altamira, Fundación OSDE

Elgue, M. C. (2003). El sentido del Desarrollo y la Economía Social. Buenos Aires: FLACSO Facultad Latinoamericana de Ciencias Sociales Sede Académica Argentina

Elgue, M. C. (2007). La Economía Social. Fundación Centro de Investigaciones de la Economía Social (CIESO). Buenos Aires: Ed. Capital Intelectual

Fonteneau, B. (2010). "Economía social y solidaria: construyendo un entendimiento común". Documento de trabajo 2010. CIF-OIT, Turín, Italia

Gambina, J. C. \& Elorza, E. (2013) (Comps.). La economía política en el debate de las ciencias sociales. San Luis: Nueva Editorial Universitaria.

Gandulfo, A. (2011). Construcción de la Política Pública: Programa Nacional de Microcrédito en Argentina En Kliksberg, Bernardo (Comp.). América Latina frente a la Crisis. Buenos Aires: Editorial Sudamericana.

Hidalgo, A. (2014). Análisis de la Implementación de CONAMI en la ciudad de San Luis. Aproximaciones a las problemáticas de la ejecución de Políticas Sociales. Tesina de Especialización en Gestión Social, Facultad de Ciencias Políticas y Sociales, Universidad Nacional de Cuyo.

Hintze, S. (2010) "La Política es un arma cargada de futuro. La economía social y solidaria en Brasil y Venezuela" Colección Becas de Investigación. Clacso. Noviembre de 2010

Iazzeta, O. La reconstrucción del Estado: Una mirada desde la democracia". Rev. Latinoamericana de Política Social N N 2. Pp. 33-52

Kaztman, R. (2001). Seducidos y abandonados: el aislamiento social de los pobres urbanos., Revista de la Cepal, № 75. Pp. 171-189

Ley 26117 "Promoción del Microcrédito para el Desarrollo de la Economía Social". 
Madoery, O. (2013). Tres tesis para una re-interpretación política del desarrollo. Revista Temas y Debates. ISSN 1666-0714, número 26, pp. 13-37.

Martínez Nogueira, R. (2007). Desafíos estratégicos en la implementación de programas sociales. En Cortázar Velarde, Juan Carlos (ed.) Entre el diseño y la evaluación. El papel crucial de la implementación de los programas sociales. Washington, D.C.: Banco Interamericano de Desarrollo.

Paime, J. (2000). La Modernización de la Protección Social en Europa en Revista Latinoamericana de Política Social. No 2. Pp. 9-32. Rofman y Vásquez Blanco. (2011). Al cierre del Bicentenario dos modelos de país en disputa. En Vázquez Blanco, J.M y Fraschina, S. (comps.) (2011) Aportes de la Economía Política en el Bicentenario. Buenos Aires: Prometeo Libros,

Roitman, R. (2011). "Estudio de caracterización y cuantificación de la Economía Social Argentina". Primera Fase. Convenio Instituto Nacional de Asociativismo y Economía Social y Foro de Ciencia y Tecnología para la Producción.

Roitman, R. \& Cabonetto, S. (2005) (comp. c/ Billone J, Aprile H, y Carbonetto, S) "El microcrédito: una herramienta para la inclusión social", contribuciones de la Mesa Federal de Entidades de Promoción del Desarrollo de la Economía Social, Bs. As

Organización Internacional del Trabajo (2002). R193 Recomendación sobre la promoción de las cooperativas (núm. 193) Recuperado de http://www.ilo.org/dyn/normlex/es/f?p=NORMLEXPUB:12100:0::NO::P12100 INSTRU MENT ID:312531

Santos, Boaventura De Sousa. (2000). Crítica de la Razón Indolente. Bilbao: Editorial Desclée de Brouwer.

Servaes, J. (2000). Comunicación para el Desarrollo: tres paradigmas, dos modelos, en Revista Temas y Problemas. Vol. 10. Pp. 5-28 